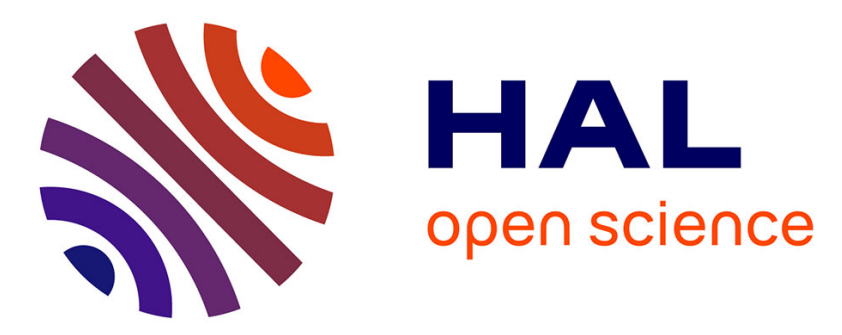

\title{
Trajectory tracking fault tolerant controller design for Takagi-Sugeno systems subject to actuator faults
}

\author{
Tahar Bouarar, Benoît Marx, Didier Maquin, José Ragot
}

\section{To cite this version:}

Tahar Bouarar, Benoît Marx, Didier Maquin, José Ragot. Trajectory tracking fault tolerant controller design for Takagi-Sugeno systems subject to actuator faults. International Conference on Communications, Computing and Control Applications, CCCA'11, Mar 2011, Hammamet, Tunisia. pp.CDROM, 10.1109/CCCA.2011.6031484 . hal-00567360

\section{HAL Id: hal-00567360 https://hal.science/hal-00567360}

Submitted on 8 Apr 2014

HAL is a multi-disciplinary open access archive for the deposit and dissemination of scientific research documents, whether they are published or not. The documents may come from teaching and research institutions in France or abroad, or from public or private research centers.
L'archive ouverte pluridisciplinaire HAL, est destinée au dépôt et à la diffusion de documents scientifiques de niveau recherche, publiés ou non, émanant des établissements d'enseignement et de recherche français ou étrangers, des laboratoires publics ou privés. 


\title{
Trajectory tracking fault tolerant controller design for Takagi-Sugeno systems subject to actuator faults
}

\author{
Tahar Bouarar, Benoît Marx, Didier Maquin, José Ragot
}

\begin{abstract}
This paper investigates the problem of fault tolerant control (FTC) design for nonlinear Takagi-Sugeno (T-S) models with measurable premise variables. The idea is to synthesize a fault tolerant controller ensuring state trajectory tracking. Based on Lyapunov theory, new less conservative approaches are proposed in term of Linear Matrix Inequality (LMI). A PI observer is needed to estimate simultaneously the faults and the faulty system states in order to reconfigure the FTC law. A numerical example is considered to compare the conservatism of the proposed FTC approaches with the existing one and to illustrate the effectiveness of the FTC technique vs. the classical controller design methodology.
\end{abstract}

Keywords- Takagi-Sugeno nonlinear models, PI observer, state and fault estimation, LMI, Lyapunov theory, $L_{2}$ norm.

\section{INTRODUCTION}

The classical control law schemes have shown their interest in the system stabilization framework. Nevertheless, if faults affect the system, the classical controllers may not ensure the system stabilization. In this case, fault tolerant control is introduced to take into account the faults affecting the system components. In literature, two kinds of strategies dealing with the above problem have been proposed. The first one is called robust control or passive FTC. The main idea of this technique is to consider the faults as non structural bounded uncertainties which effect on the system will be minimized by using the $L_{2}$ norm. The passive control strategy is designed only for norm bounded faults which constitutes a major drawback of this technique. The second kind is called active FTC strategy. This latter requires the knowledge of the faults to reconfigure the controller to ensure the stability of the faulty system.

The FTC problem has already been studied in the literature. For instance, fault tolerant controller design methodology for linear systems is proposed by [1], [2], [3], [4] and [5]. Recently, this study has been extended to the nonlinear systems given in Takagi-Sugeno [6] representation by [8]. Nevertheless, the proposed approach may be conservative. Moreover, new approaches for trajectory tracking FTC design for T-S models with unmeasurable premise variables have been proposed by [7] and [9].

This paper aims to reduce the conservatism of the results proposed in [8] and to show the effectiveness of the FTC law compared to a classical one when faults affect the system dynamics. Thus, this paper is organized as follows. In the next section, the problem of fault tolerant controller design is presented. In section 3, an active FTC approach is proposed. In the last section, a numerical example is considered to illustrate the efficiency of the proposed active FTC approach compared to a passive one (developed in the appendix). Moreover, the

All the authors are with Centre de Recherche en Automatique de Nancy (CRAN). Nancy-Université CNRS, 2, avenue de la forêt de Haye, 54516 Vandoeuvre-lès-Nancy. \{tahar.bouarar, benoit.marx, didier.maquin, jose.ragot\}@ ensem.inpl-nancy.fr feasibility areas of the proposed active FTC approach and the one given in [8] are compared.

The following notations are considered to improve the paper readability. The single or double sums can be rewritten as:

$$
\phi_{\mu}=\sum_{i=1}^{r} \mu_{i}(\xi(t)) \phi_{i} \text { and } \phi_{\mu \mu}=\sum_{i=1}^{r} \sum_{j=1}^{r} \mu_{i}(\xi(t)) \mu_{j}(\xi(t)) \phi_{i j} .
$$

The symbol $*$ denotes the transposed element in the symmetric positions of a matrix and $\operatorname{diag}\left(M_{1}, \ldots, M_{r}\right)$ is a block diagonal matrix which diagonal entries are defined by $M_{1}, \ldots, M_{r}$. The following lemma will be needed.

Lemma 1 [10]: Consider two real matrices $X$ and $Y$ with appropriate dimensions, for any positive scalar $\delta$ the following inequality holds:

$$
X^{T} Y+Y^{T} X \leq \delta X^{T} X+\delta^{-1} Y^{T} Y
$$

\section{PROBLEM FORMULATION}

Let us consider the following T-S model without faults corresponding to the reference model.

$$
\left\{\begin{array}{l}
\dot{x}(t)=\sum_{i=1}^{r} \mu_{i}(\xi(t))\left(A_{i} x(t)+B_{i} u(t)\right) \\
y(t)=\sum_{i=1}^{r} \mu_{i}(\xi(t))\left(C_{i} x(t)+D_{i} u(t)\right)
\end{array}\right.
$$

where $r$ is the number of submodels, $\xi(t)$ is the measurable premise variable, $\mu_{i}(\xi(t))$ are the membership functions verifying the convex sum property $0 \leq \mu_{i}(\xi(t)) \leq 1$ and $\sum_{i=1}^{r} \mu_{i}(\xi(t))=1, \quad x(t) \in \mathbb{R}^{n}, \quad y(t) \in \mathbb{R}^{p} \quad$ and $\quad u(t) \in \mathbb{R}^{m}$ represent respectively the state, the output and the input vectors, $\left\{A_{i}, B_{i}, C_{i}, D_{i}\right\}$ are the submodels matrices. Consider the faulty system given by

$$
\left\{\begin{array}{l}
\dot{x}_{f}(t)=\sum_{i=1}^{r} \mu_{i}(\xi(t))\left(A_{i} x_{f}(t)+B_{i}\left(u_{f}(t)+f(t)\right)\right) \\
y_{f}(t)=\sum_{i=1}^{r} \mu_{i}(\xi(t))\left(C_{i} x_{f}(t)+D_{i}\left(u_{f}(t)+f(t)\right)\right)
\end{array}\right.
$$

where $x_{f}(t) \in \mathbb{R}^{n}, \quad y_{f}(t) \in \mathbb{R}^{p}$ and $u_{f}(t) \in \mathbb{R}^{m}$ represent respectively the faulty state and faulty output vectors and the fault tolerant control signal, $f(t) \in \mathbb{R}^{m}$ is the fault directly 
affecting the input. The fault is supposed to be constant (i.e. $d f(t) / d t=0)$.

The objective is to design a fault tolerant controller ensuring the convergence of the faulty state vector $x_{f}(t)$ to the nominal one $x(t)$. The methodology of controller conception is based on the scheme depicted in Fig.1.

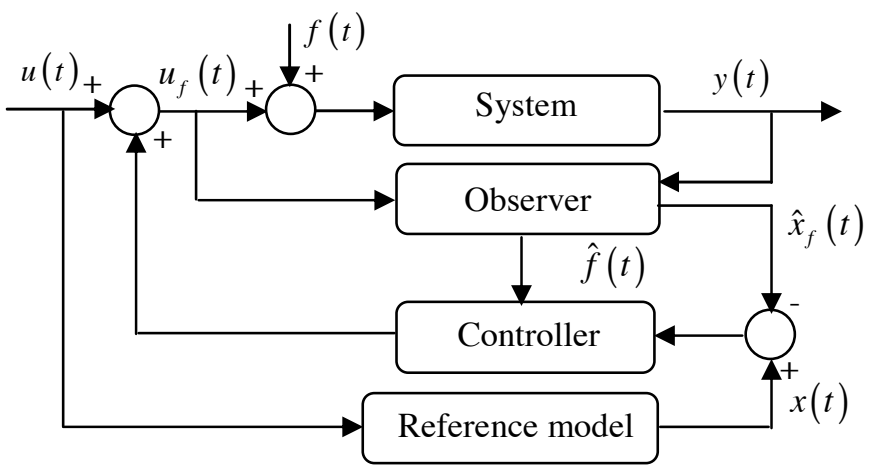

Fig.1. Fault tolerant control strategy

Let us consider the FTC law given by:

$$
u_{f}(t)=\sum_{i=1}^{r} \mu_{i}(\xi(t)) K_{i}\left(x(t)-\hat{x}_{f}(t)\right)+u(t)-\hat{f}(t)
$$

where: $K_{i} \in \mathbb{R}^{m \times n}$ are the state feedback gain matrices to be synthesized. The FTC design simultaneously requires the knowledge of the faulty state vector and the faults affecting the system. In order to estimate $x_{f}(t)$ and $f(t)$, the following PI observer is considered:

$$
\left\{\begin{array}{l}
\dot{\hat{x}}_{f}(t)=\sum_{i=1}^{r} \mu_{i}(\xi(t))\left(A_{i} \hat{x}_{f}(t)+B_{i}\left(u_{f}(t)+\hat{f}(t)\right)+H_{i}^{1}\left(y_{f}(t)-\hat{y}_{f}(t)\right)\right) \\
\dot{\hat{f}}(t)=\sum_{i=1}^{r} \mu_{i}(\xi(t))\left(H_{i}^{2}\left(y_{f}(t)-\hat{y}_{f}(t)\right)\right) \\
\hat{y}_{f}(t)=\sum_{i=1}^{r} \mu_{i}(\xi(t))\left(C_{i} \hat{x}_{f}(t)+D_{i}\left(u_{f}(t)+\hat{f}(t)\right)\right)
\end{array}\right.
$$

where $H_{i}^{1} \in \mathbb{R}^{n \times p}$ and $H_{i}^{2} \in \mathbb{R}^{m \times p}$ are the observer's gain matrices to be determined to estimate $f(t)$ and $x_{f}(t)$. A first solution to this problem was proposed in theorem 5.4 of [8].

\section{FAULT TOLERANT CONTROLLER DESIGN}

In this section we propose a less conservative approach for fault tolerant controller conception. Let us respectively define the state and fault estimation errors defined by: $e_{s}(t)=x_{f}(t)-\hat{x}_{f}(t)$ and $e_{d}(t)=f(t)-\hat{f}(t)$. Let us also define the state tracking error $e_{p}(t)=x(t)-x_{f}(t)$ and the output estimation error $e_{y}(t)=y_{f}(t)-\hat{y}_{f}(t)$. By adding and substracting $K_{\mu} x_{f}(t)$ in (4), one can obtain:
$u_{f}(t)=K_{\mu}\left(x(t)-x_{f}(t)\right)+K_{\mu}\left(x_{f}(t)-\hat{x}_{f}(t)\right)+u(t)-\hat{f}(t)(6)$

The dynamics of $e_{p}(t)$ and $e_{s}(t)$ are given by:

$$
\begin{gathered}
\dot{e}_{p}(t)=\left(A_{\mu}-B_{\mu} K_{\mu}\right) e_{p}(t)-B_{\mu} K_{\mu} e_{s}(t)-B_{\mu} e_{d}(t) \\
\dot{e}_{s}(t)=A_{\mu} e_{s}(t)+B_{\mu} e_{d}(t)-H_{\mu}^{1} e_{y}(t)
\end{gathered}
$$

According to (8), to avoid the crossing terms resulting from the observer's gains $H_{i}^{1}$ and system matrices ( $C_{i}$ and $D_{i}$ ) multiplication, we introduce a "virtual dynamics" in the output error $e_{y}(t)$ [11] [12]. This latter can be expressed as:

$$
0 \dot{e}_{y}(t)=C_{\mu} e_{s}(t)+D_{\mu} e_{d}(t)-e_{y}(t)
$$

where $0 \in \mathbb{R}^{p \times p}$ is a zero matrix.

Since the faults affecting the system are supposed to be constant (i.e. $\dot{f}(t)=0$ ), the dynamics of the fault estimation error is given by:

$$
\dot{e}_{d}(t)=-H_{\mu}^{2} C_{\mu} e_{s}(t)-H_{\mu}^{2} D_{\mu} e_{d}(t)
$$

The combination of (7), (8), (9) and (10) allows the formulation of the dynamics of $e_{y}(t), e_{p}(t), e_{s}(t)$ and $e_{d}(t)$ in a descriptor form:

$$
E \dot{\tilde{e}}(t)=\tilde{A}_{\mu} \tilde{e}(t)
$$

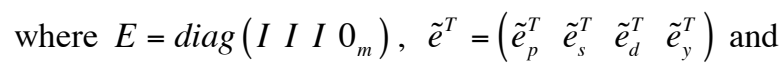

$$
\tilde{A}_{\mu}=\left(\begin{array}{cccc}
A_{\mu}-B_{\mu} K_{\mu} & -B_{\mu} K_{\mu} & -B_{\mu} & 0 \\
0 & A_{\mu} & B_{\mu} & -H_{\mu}^{1} \\
0 & -H_{\mu}^{2} C_{\mu} & -H_{\mu}^{2} D_{\mu} & 0 \\
0 & C_{\mu} & D_{\mu} & -I
\end{array}\right)
$$

The main proposed result can now be established.

Theorem 1: The tracking error $e_{p}(t)$, the state $e_{s}(t)$ and fault $e_{d}(t)$ estimation errors asymptotically converge to zero if there exists some matrices $X=X^{T} \geq 0, P_{6}=P_{6}^{T} \geq 0, P_{11}=I$, $P_{13}, P_{14}, P_{15}, P_{16}, \bar{H}_{i}^{1}, \bar{H}_{j}^{2}$ and $K_{j}$ such that the following LMI are satisfied for all $i, j=1,2, . ., r$ :

$$
\left(\begin{array}{cc}
\Upsilon_{i j}^{(1,1)} & * \\
\Upsilon_{i}^{(2,1)} & \Upsilon^{(2,2)}
\end{array}\right)<0
$$

where:

$$
Y_{i j}^{(1,1)}=\left(\begin{array}{cccccc}
A_{i} X+X A_{i}^{T} & * & * & 0 & * & * \\
-K_{j}^{T} B_{i}^{T} & \Sigma_{i}^{(2,2)} & * & * & 0 & 0 \\
-B_{i}^{T} & \Sigma_{i j}^{(3,2)} & \Sigma_{i j}^{(3,3)} & * & 0 & 0 \\
0 & \Sigma_{i}^{(4,2)} & \Sigma_{i}^{(4,3)} & -P_{16}-P_{16}^{T} & 0 & 0 \\
K_{j}^{T} B_{i}^{T} & 0 & 0 & 0 & -I & 0 \\
X & 0 & 0 & 0 & 0 & -I
\end{array}\right)
$$




$$
\begin{aligned}
& \Upsilon_{i}^{(2,1)}=\left(\begin{array}{cccccc}
X & 0 & 0 & 0 & 0 & 0 \\
0 & P_{13}^{T} C_{i} & 0 & 0 & 0 & 0 \\
X & 0 & 0 & 0 & 0 & 0 \\
0 & 0 & P_{13}^{T} D_{i} & 0 & 0 & 0 \\
X & 0 & 0 & 0 & 0 & 0 \\
0 & 0 & 0 & P_{13}^{T} & 0 & 0
\end{array}\right) \\
& \Upsilon^{(2,2)}=\operatorname{diag}(-I \quad-I \quad-I \quad-I \quad-I \quad-I) \\
& \Sigma_{i}^{(2,2)}=P_{6} A_{i}+A_{i}^{T} P_{6}+P_{14}^{T} C_{i}+C_{i}^{T} P_{14} \\
& \Sigma_{i j}^{(3,2)}=P_{15}^{T} C_{i}-\bar{H}_{j}^{2} C_{i}+B_{i}^{T} P_{6}+D_{i}^{T} P_{14} \\
& \Sigma_{i j}^{(3,3)}=D_{i}^{T} P_{15}-\bar{H}_{j}^{2} D_{i}-D_{i}^{T}\left(\bar{H}_{j}^{2}\right)^{T} \\
& \Sigma_{i}^{(4,2)}=P_{16}^{T} C_{i}-\bar{H}_{i}^{1}-P_{14} \\
& \Sigma_{i}^{(4,3)}=P_{16}^{T} D_{i}-P_{15} \text {. }
\end{aligned}
$$

Proof: Let us consider the following candidate quadratic Lyapunov function:

$$
V(\tilde{e}(t))=\tilde{e}^{T}(t) E P \tilde{e}(t)
$$

with:

$$
E P=P^{T} E \geq 0
$$

A way to provide easily LMI conditions is to consider the matrix $P$ structure as follows:

$$
P=\left(\begin{array}{cccc}
P_{1} & 0 & 0 & 0 \\
0 & P_{6} & 0 & 0 \\
0 & 0 & P_{11} & 0 \\
P_{13} & P_{14} & P_{15} & P_{16}
\end{array}\right)
$$

According to (15), it follows that $P_{1}=P_{1}^{T} \geq 0, P_{6}=P_{6}^{T} \geq 0$, $P_{11}=P_{11}^{T} \geq 0$ and $P_{13}, P_{14}, P_{15}, P_{16}$ are free slack matrices.

The tracking error $e_{p}(t)$, the state $e_{s}(t)$ and the fault $e_{d}(t)$ estimation errors converge asymptotically to zero if:

$$
\dot{V}(\tilde{e}(t))=\dot{\tilde{e}}^{T}(t) E P \tilde{e}(t)+\tilde{e}^{T}(t) E P \dot{\tilde{e}}(t)<0
$$

With (11) and (14), the inequality (17) becomes:

$$
\tilde{e}^{T}(t)\left(\tilde{A}_{\mu}^{T} P+P^{T} \tilde{A}_{\mu}\right) \tilde{e}(t)<0
$$

The inequality (18) is fulfilled if:

$$
\tilde{A}_{\mu}^{T} P+P^{T} \tilde{A}_{\mu}<0
$$

Indeed, with (12) and (16) the inequality (19) becomes:

$$
\left(\begin{array}{cccc}
\bar{\Psi}_{\mu \mu}^{(1,1)} & * & * & * \\
C_{\mu}^{T} P_{13}-K_{\mu}^{T} B_{\mu}^{T} P_{1} & \bar{\Psi}_{\mu}^{(2,2)} & * & * \\
D_{\mu}^{T} P_{13}-B_{\mu}^{T} P_{1} & \bar{\Psi}_{\mu \mu}^{(3,2)} & \bar{\Psi}_{\mu \mu}^{(3,3)} & * \\
-P_{13} & \bar{\Psi}_{\mu}^{(4,2)} & P_{16}^{T} D_{\mu}-P_{15} & -P_{16}-P_{16}^{T}
\end{array}\right)<0(20)
$$

where: $\bar{\Psi}_{\mu \mu}^{(1,1)}=P_{1} A_{\mu}+A_{\mu}^{T} P_{1}-P_{1} B_{\mu} K_{\mu}-K_{\mu}^{T} B_{\mu}^{T} P_{1}$

$\bar{\Psi}_{\mu}^{(2,2)}=P_{14}^{T} C+C_{\mu}^{T} P_{14}+P_{6} A_{\mu}+A_{\mu}^{T} P_{6}$

$\bar{\Psi}_{\mu \mu}^{(3,2)}=B_{\mu}^{T} P_{6}-P_{11} H_{\mu}^{2} C_{\mu}+P_{15}^{T} C_{\mu}+D_{\mu}^{T} P_{14}$

$\bar{\Psi}_{\mu \mu}^{(3,3)}=P_{15}^{T} D_{\mu}+D_{\mu}^{T} P_{15}-P_{11} H_{\mu}^{2} D_{\mu}-D_{\mu}^{T}\left(H_{\mu}^{2}\right)^{T} P_{11}$

$\bar{\Psi}_{\mu}^{(4,2)}=P_{16}^{T} C_{\mu}-\left(H_{\mu}^{1}\right)^{T} P_{6}-P_{14}$.

Multiplying (20) left and right by $\operatorname{diag}(X I I I I)$ where $X=P_{1}^{-1}$, and considering $P_{11}=P_{11}^{T}=I>0$ and the bijective variable changes $\left(H_{\mu}^{1}\right)^{T} P_{6}=\bar{H}_{\mu}^{1}, P_{11} H_{\mu}^{2}=\bar{H}_{\mu}^{2}$, (20) yields:

$$
\left(\begin{array}{cccc}
\Phi_{\mu \mu}^{(1,1)} & * & * & * \\
C_{\mu}^{T} P_{13} X-K_{\mu}^{T} B_{\mu}^{T} & \bar{\Psi}_{\mu}^{(2,2)} & * & * \\
D_{\mu}^{T} P_{13} X-B_{\mu}^{T} & \Phi_{\mu \mu}^{(3,2)} & \Phi_{\mu \mu}^{(3,3)} & * \\
-P_{13} X & \Phi_{\mu}^{(4,2)} & P_{16}^{T} D_{\mu}-P_{15} & -P_{16}-P_{16}^{T}
\end{array}\right)<0(21)
$$

where:

$\Phi_{\mu \mu}^{(1,1)}=A_{\mu} X+X A_{\mu}^{T}-B_{\mu} K_{\mu} X-X K_{\mu}^{T} B_{\mu}^{T}$

$\Phi_{\mu \mu}^{(3,2)}=B_{\mu}^{T} P_{6}-\bar{H}_{\mu}^{2} C_{\mu}+P_{15}^{T} C_{\mu}+D_{\mu}^{T} P_{14}$

$\Phi_{\mu \mu}^{(3,3)}=P_{15}^{T} D_{\mu}+D_{\mu}^{T} P_{15}-\bar{H}_{\mu}^{2} D_{\mu}-D_{\mu}^{T}\left(\bar{H}_{\mu}^{2}\right)^{T}$

$\Phi_{\mu}^{(4,2)}=P_{16}^{T} C_{\mu}-\bar{H}_{\mu}^{1}-P_{14}$

Applying lemma 1 and considering $\delta_{1}=\delta_{2}=\delta_{3}=\delta_{4}=I$, the inequality (21) is implied by:

$$
\left(\begin{array}{cccc}
\bar{\Phi}_{\mu \mu}^{(1,1)} & * & * & 0 \\
-K_{\mu}^{T} B_{\mu}^{T} & \bar{\Phi}_{\mu \mu}^{(2,2)} & * & * \\
-B_{\mu}^{T} & \Phi_{(3,2)} & \bar{\Phi}_{\mu \mu}^{(3,3)} & * \\
0 & P_{16}^{T} C_{\mu}-\bar{H}_{\mu}^{1}-P_{14} & P_{16}^{T} D_{\mu}-P_{15} & \bar{\Phi}^{(4,4)}
\end{array}\right)<0(22)
$$

where:

$$
\begin{aligned}
& \bar{\Phi}_{\mu \mu}^{(1,1)}=\left(\begin{array}{l}
A_{\mu} X+X A_{\mu}^{T}+\delta_{1} B_{\mu} K_{\mu} K_{\mu}^{T} B_{\mu}^{T}+\delta_{1}^{-1} X X \\
+\delta_{2}^{-1} X X+\delta_{3}^{-1} X X+\delta_{4}^{-1} X X
\end{array}\right) \\
& \bar{\Phi}_{\mu \mu}^{(2,2)}=P_{14}^{T} C+C_{\mu}^{T} P_{14}+P_{6} A_{\mu}+A_{\mu}^{T} P_{6}+\delta_{2} C_{\mu}^{T} P_{13} P_{13}^{T} C_{\mu} \\
& \bar{\Phi}_{\mu \mu}^{(3,3)}=D_{\mu}^{T} P_{15}-\bar{H}_{\mu}^{2} D_{\mu}-D_{\mu}^{T}\left(\bar{H}_{\mu}^{2}\right)^{T}+\delta_{3} D_{\mu}^{T} P_{13} P_{13}^{T} D_{\mu} \\
& \bar{\Phi}^{(4,4)}=\delta_{4} P_{13} P_{13}^{T}-P_{16}-P_{16}^{T} .
\end{aligned}
$$

Applying Schur complement [13] on the BMI terms $\bar{\Phi}_{\mu \mu}^{(1,1)}$, $\bar{\Phi}_{\mu \mu}^{(2,2)}$ and $\bar{\Phi}_{\mu \mu}^{(3,3)}$, the sufficient LMI conditions proposed in the theorem 1 hold.

Remark 1: New LMI conditions can be provided from the ones given in theorem 1 by considering only the diagonal matrices 
of (16) (i.e. $P_{1}, P_{6}, P_{11}$ and $P_{16}$ ). This result is given in corollary 1 .

Corollary 1: The tracking error $e_{p}(t)$, the state $e_{s}(t)$ and the fault $e_{d}(t)$ estimation errors convergence asymptotically to zero if there exists the matrices $X=X^{T} \geq 0, P_{6}=P_{6}^{T} \geq 0$, $P_{11}=I, P_{16}, \bar{H}_{i}^{1}, \bar{H}_{j}^{2}$ and $K_{\mu}$ such that the following LMI are satisfied for all $i, j=1,2, . ., r$

$\left(\begin{array}{cccccc}\Theta_{i}^{(1,1)} & * & * & 0 & * & * \\ -K_{j}^{T} B_{i}^{T} & \Theta_{i}^{(2,2)} & * & * & 0 & 0 \\ -B_{i}^{T} & \Theta_{i}^{(3,2)} & \Theta_{i j}^{(3,3)} & * & 0 & 0 \\ 0 & P_{16}^{T} C_{i}-\bar{H}_{i}^{1} & P_{16}^{T} D_{i} & -P_{16}-P_{16}^{T} & 0 & 0 \\ K_{j}^{T} B_{i}^{T} & 0 & 0 & 0 & -I & 0 \\ X & 0 & 0 & 0 & 0 & -I\end{array}\right)<0(23)$

where: $\Theta_{i}^{(1,1)}=A_{i} X+X A_{i}^{T}, \Theta_{i}^{(2,2)}=P_{6} A_{i}+A_{i}^{T} P_{6}$, $\Theta_{i}^{(3,2)}=B_{i}^{T} P_{6}-\bar{H}_{j}^{2} C_{i}$ and $\Theta_{i j}^{(3,3)}=-\bar{H}_{j}^{2} D_{i}-D_{i}^{T}\left(\bar{H}_{j}^{2}\right)^{T}$.

Remark 2: To ensure the stability of (3) even if faults occur, one has to check the existence of diag $\left(\mathrm{X}_{2} I\right)$ in theorem 5.4 of [8] or the matrix $P$ given by (16) in the proposed approach. Indeed, the proposed approach (theorem 1) introduces some additional free slack variables to relax the existing LMI conditions. This conservatism reduction can be shown mathematically by considering in theorem 1 that $P_{13}=0, P_{14}=0, P_{15}=0$ and $P_{16}=0$. Then, the inequality (22) can be rewritten as:

$$
\left(\begin{array}{cccc}
\bar{\Phi}_{\mu \mu}^{(1,1)} & * & * & 0 \\
-K_{\mu}^{T} B_{\mu}^{T} & \bar{\Phi}_{\mu}^{(2,2)} & * & * \\
-B_{\mu}^{T} & \Phi_{\mu \mu}^{(3,2)} & \bar{\Phi}_{\mu \mu}^{(3,3)} & 0 \\
0 & -\bar{H}_{\mu}^{1} & 0 & 0
\end{array}\right)<0
$$

with $\bar{\Phi}_{\mu \mu}^{(1,1)}=A_{\mu} X+X A_{\mu}^{T}+\delta_{1} B_{\mu} K_{\mu} K_{\mu}^{T} B_{\mu}^{T}+\delta_{1}^{-1} X X$, $\bar{\Phi}_{\mu}^{(2,2)}=P_{6} A_{\mu}+A_{\mu}^{T} P_{6}$ and $\bar{\Phi}_{\mu \mu}^{(3,3)}=-\bar{H}_{\mu}^{2} D_{\mu}-D_{\mu}^{T}\left(\bar{H}_{\mu}^{2}\right)^{T}$.

Replacing $e_{y}(t)$ by its expression given in (24), one obtains the LMI conditions of theorem 5.4 [8].

\section{SIMULATION RESULTS}

In order to show the effectiveness and the applicability of the proposed approaches, let us consider the system (2) with

$$
\begin{aligned}
& A_{1}=\left(\begin{array}{ccc}
a & 1 & 1 \\
1 & -3 & 0 \\
2 & 1 & -8
\end{array}\right), A_{2}=\left(\begin{array}{ccc}
-3 & 2 & 2 \\
0 & -3 & 0.2 \\
0.5 & 2 & -5
\end{array}\right), B_{1}=\left(\begin{array}{c}
0 \\
1 \\
0.25
\end{array}\right), \\
& B_{2}=\left(\begin{array}{l}
1 \\
1 \\
b
\end{array}\right), C_{1}=\left(\begin{array}{c}
-1 \\
0.5 \\
0
\end{array}\right)^{T}, C_{2}=\left(\begin{array}{c}
-1 \\
-0.5 \\
0
\end{array}\right)^{T}, D_{1}=-0.8
\end{aligned}
$$

$D_{2}=-0.5 \quad, \quad \mu_{1}(u(t))=\frac{1-\tanh (0.5-u(t))}{2} \quad$ and $\mu_{2}(u(t))=1-\mu_{1}(u(t)) . a$ and $b$ are two model parameters. Firstly, our aim is to compare the conservatism of the approach given in theorem 5.4 of [8] and the proposed theorem 1 and corollary 1 .

Let us consider $a \in\left[\begin{array}{ll}-2 & -0.6\end{array}\right]$ and $b \in\left[\begin{array}{ll}-2 & 0\end{array}\right]$, using Matlab LMI Toolbox the obtained feasibility fields are

\begin{tabular}{|c|c|c|c|c|c|c|c|c|c|c|c|c|c|}
\hline & • & • & • & • & • & • & • & • & • & • & • & • & \\
\hline$-0.2 t$ & . & • & 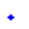 & • & . & * & . & • & - & . & $\bullet$ & $\cdot$ & \\
\hline & 图 & 图 & 圈 & 图 & 图 & 因 & * & * & * & * & * & * & \\
\hline-0.4 東 & 图 & 图 & 国 & 图 & 因 & 图 & 因 & * & * & * & * & * & $\bullet$ \\
\hline & 圈 & 图 & 国 & 圈 & 圈 & 园 & 图 & $*$ & * & * & * & * & 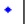 \\
\hline-0.6 雨 & 图 & 图 & 图 & 图 & 圈 & 图 & 图 & 图 & * & * & * & * & \\
\hline & 图 & 图 & 图 & 图 & 图 & 图 & 圈 & 图 & * & * & * & * & \\
\hline 8雨 & 图 & 图 & 图 & 圈 & 圈 & 图 & 图 & 图 & * & * & * & * & $*$ \\
\hline & 圈 & 圈 & 图 & 图 & 图 & 图 & 图 & 图 & * & $*$ & * & $*$ & \\
\hline ـ -1 本 & 圈 & 图 & 图 & 图 & 图 & 图 & 图 & 图 & * & * & * & * & \\
\hline 雨 & 圈 & 图 & 图 & 圈 & 图 & 图 & 国 & 图 & * & * & * & * & * \\
\hline-1.2 東 & 图 & 图 & 图 & 图 & 图 & 园 & 图 & 图 & * & * & * & * & \\
\hline & 图 & 图 & 图 & 图 & 图 & 图 & 图 & 图 & * & * & * & * & * \\
\hline-1.4 雨 & 图 & 图 & 困 & 图 & 图 & 图 & 图 & 图 & * & * & * & * & *- \\
\hline & 图 & 圆 & 园 & 图 & 图 & 因 & 图 & 图 & * & * & * & * & * \\
\hline -1.6座 & 圈 & 图 & 图 & 图 & 图 & 图 & 图 & 图 & * & * & * & * & \\
\hline & 圈 & 图 & 图 & 图 & 图 & 图 & 图 & 图 & * & * & * & * & * \\
\hline-1.8 東 & 图 & 图 & 图 & 图 & 图 & 图 & 图 & 图 & * & * & * & $*$ & $k$ \\
\hline 東 & 图 & 图 & 图 & 图 & 图 & 图 & 图 & 图 & * & * & * & * & \\
\hline-2 & & . & & 1.6 & & -1.4 & & -1.2 & & 1 & & 1.8 & \\
\hline
\end{tabular}
presented in Fig. 2 and show that the proposed approaches are less conservative than in [8].

Fig.2. Feasibility fields ; - Theorem $1, \times$ Corollary 1 and $\square$ Theorem 5.4 of [8]

Secondly, in order to illustrate the effectiveness of the fault tolerant controller compared to a classical one, a passive FTC controller is designed as described in appendix, in order to minimize the $L_{2}$-gain from the fault to the tracking error. The obtained results are compared with those issued from the proposed active FTC controller.

In the fault free case, it can be seen on Fig.3 that both passive and active FTC controllers ensure the system stabilization. The simulation is ran for $a=-2, b=-0.5$, a nominal input given by $u(t)=\sin (\cos (2 t) 0.5 t)$ and the LMI problem is solved with Matlab LMI Toolbox.

In order to compare passive and active FTC control facing the occurrence of a fault, a piecewise constant fault $f(t)$, occurring at $t=4$ is considered. The simulation results are displayed on the Fig. 4, 5, 6, 7. The effectiveness of the proposed FTC design can be seen on Fig.4, whereas the passive FTC fails to ensure trajectory tracking when $f(t)$ occurs.

\section{CONCLUSION}

In this paper, a trajectory tracking fault tolerant controller design approaches have been proposed for faulty T-S models with measurable premise variables. The objective is to ensure the tracking between the faulty system states and one of healthy reference model. The proposed LMI approaches are less conservative. This improvement is due to the considered "virtual dynamics" on the output error allows introducing slack variables in the Lyapunov function and decoupling the observer gains and the system matrices. 
The efficiency of the FTC law comparing with classical one is illustrated with a numerical T-S model whose input is corrected by a fault.

\section{ACKNOWLEDGMENT}

This work was supported by the GIS 3SGS.

\section{REFERENCES}

[1] Z. Gao and P.J Antsaklis, "Reconfigurable control system design via perfect model following," International Journal of Control, 56(4): 783798, 1992.

[2] M.M. Mufeed, J. Jiang and Z. Zhang, "Active fault tolerant control systems," Stochastic Analysis and Synthesis, Springer 2003.

[3] H. Noura, D. Sauter, F. Hamelin and D. Theillol, "Fault-tolerant control in dynamic systems: Application to a winding machine," IEEE Control Systems Magazine, 20(1): 33-49, 2000.

[4] B. Marx, D. Koenig and D. Georges, "Robust fault tolerant control for descriptor systems ," IEEE Transactions on Automatic Control, 49(10): 1869-1875, 2004.

[5] M. Staroswiecki, "Fault tolerant control: the pseudo-inverse method revised," 16 th IFAC World Congress, Seoul, Korea, 2005.

[6] T. Takagi and M. Sugeno, "Fuzzy identification of systems and its application to modeling and control", IEEE Trans. on System Man and Cybernetics, 15(1): 116-132. 1985.

[7] D. Ichalal, B. Marx, J. Ragot and D. Maquin, "Observer based actuator dault tolerant control in nonlinear Takagi-Sugeno fuzzy systems: LMI approach," 18th IEEE Mediterranean Conference on Control and Automation, MED'10, Marrakech, Morocco, 2010.

[8] D. Ichalal, Estimation et diagnostic de systèmes non linéaires décrits par un multimodèle de Takagi-Sugeno, PHD thesis, Nancy-Université INPL, France, 2009.

[9] D. Ichalal, B. Marx, J. Ragot and D. Maquin, "Fault tolerant control for Takagi-Sugeno systems with unmeasuable premise variables by trajectory tracking," IEEE International Symposium on Industrial Electronics, ISIE'10, Bari, Italy, 2010

[10] K. Zhou and P.P. Khargonekar, "Robust stabilization of linear systems with normbounded time-varying uncertainty," Systems and Control Letters, 10(1): 17-20, 1988.

[11] T. Bouarar, K. Guelton and N. Manamanni, "Static Output Feedback Controller Design for Takagi-Sugeno Systems - A Fuzzy Lyapunov LMI," 48th IEEE Conference on Decision and Control, CDC'09, Shanghai, China, 2009.

[12] T. Bouarar, K. Guelton and N. Manamanni, "Robust Fuzzy Lyapunov Stabilization for Uncertain and Disturbed Takagi-Sugeno Descriptors," ISA Transactions, 49(4): 447-46, 2010.

[13] S. Boyd, L. El Ghaoui, E. Féron, and V. Balakrishnan, "Linear Matrix Inequalities," System and Control Theory, Studies in Applied Mathematics, Philadelphia, PA: SIAM, 1994.

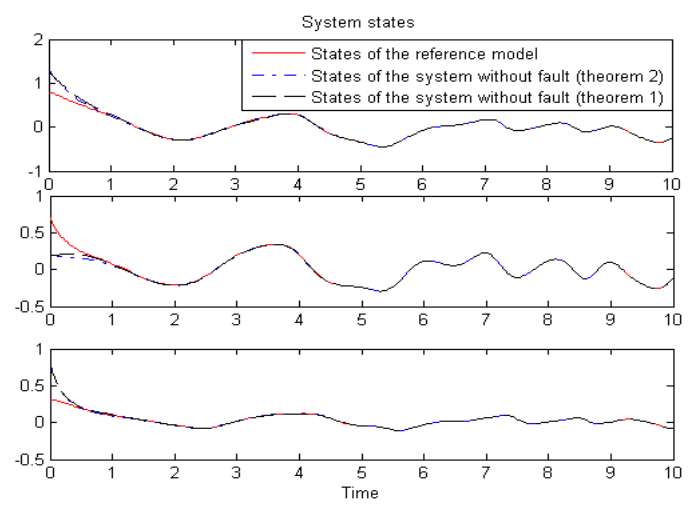

Fig.3. Comparison of the reference model states (no fault), the system states with FTC (theorem 1) and system states with classical control law (theorem 2).
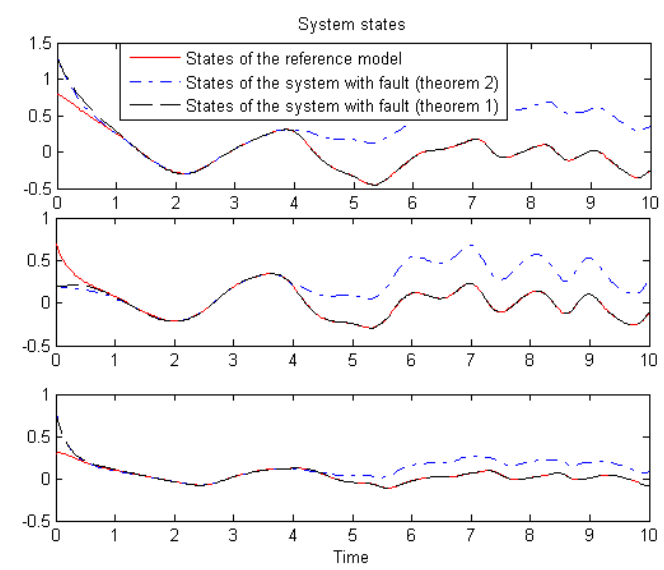

Fig.4. Comparison of the reference model state (no fault), the faulty system state with FTC (theorem 1) and the faulty system state with classical control law (theorem 2).

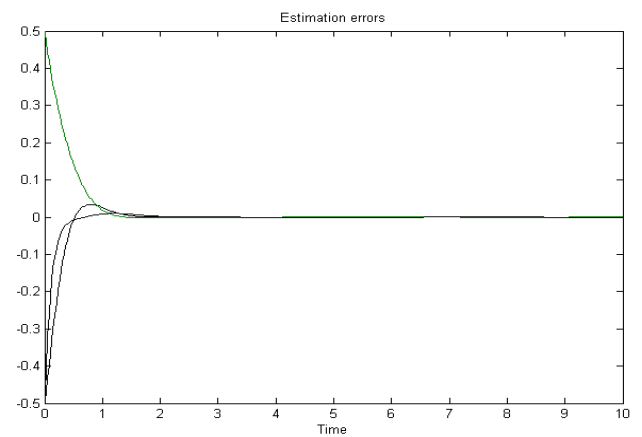

Fig.5. Estimation errors

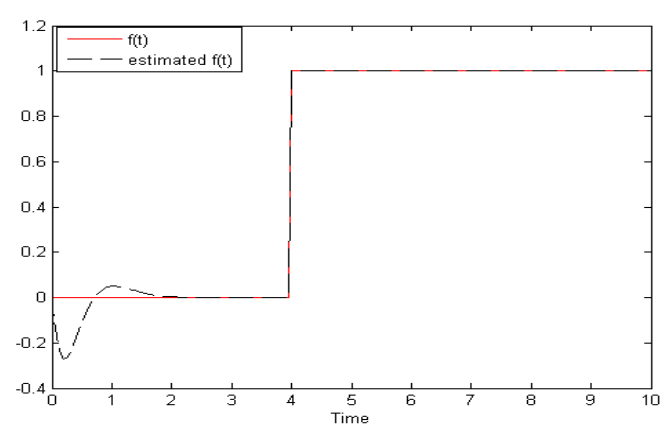

Fig.6. Fault and its estimation

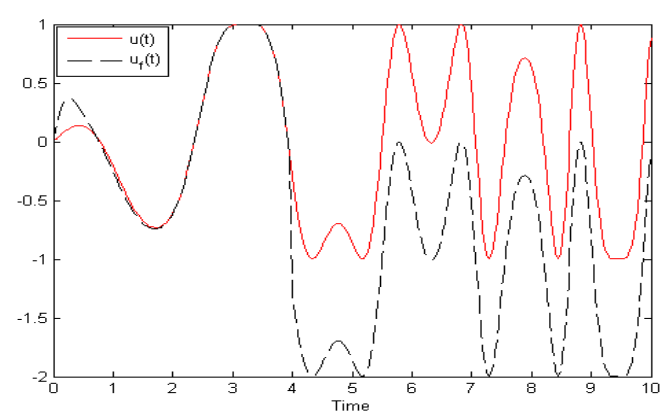

Fig.7. Nominal control input and FTC input 


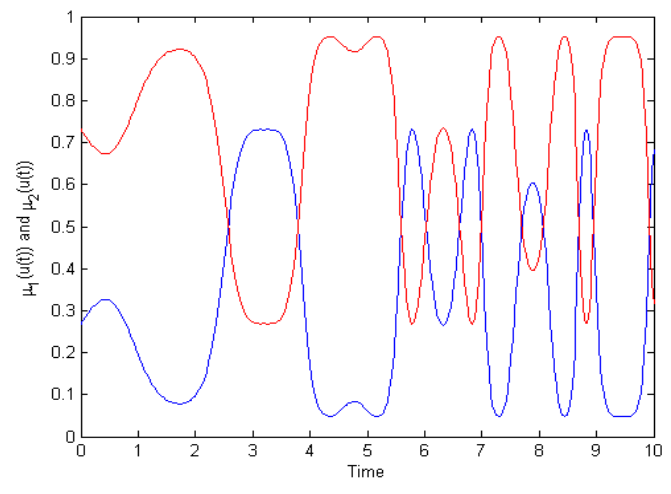

Fig.8. Membership function evolution

\section{APPENDIX. CLASSICAL CONTROLLER DESIGN APPROACH}

The classical controller design methodology is based on the following scheme.

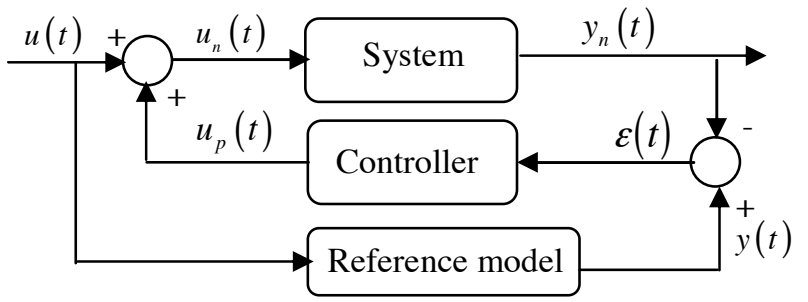

Fig.9. Classical controller design scheme

The system state representation is given by:

$$
\left\{\begin{array}{l}
\dot{x}_{n}(t)=\sum_{i=1}^{r} \mu_{i}(\xi(t))\left(A_{i} x_{n}(t)+B_{i}\left(u_{n}(t)+f(t)\right)\right) \\
y_{n}(t)=\sum_{i=1}^{r} \mu_{i}(\xi(t))\left(C_{i} x_{n}(t)+D_{i}\left(u_{n}(t)+f(t)\right)\right)
\end{array}\right.
$$

Let us define the state and output tracking errors between (2) and (25) by $e(t)=x(t)-x_{n}(t)$ and $\varepsilon(t)=y(t)-y_{n}(t)$ respectively. To ensure the tracking of the reference model, we consider the following control law $u_{p}(t)=K_{p} \varepsilon(t)$.

Introducing a "virtual dynamic" on $\varepsilon(t)$, one can obtain:

$$
\bar{E} \dot{e}(t)=\Gamma_{\mu} \bar{e}(t)-\Lambda_{\mu} f(t)
$$

where $\bar{E}=\left(\begin{array}{ll}1 & 0 \\ 0 & 0\end{array}\right), \quad \bar{e}(t)=\left(\begin{array}{c}e(t) \\ \varepsilon(t)\end{array}\right), \quad \Lambda_{\mu}=\left(\begin{array}{c}B_{\mu} \\ D_{\mu}\end{array}\right) \quad$ and $\Gamma_{\mu}=\left(\begin{array}{cc}A_{\mu} & -B_{\mu} K_{p} \\ C_{\mu} & -\left(D_{\mu} K_{p}+I\right)\end{array}\right)$.
The LMI conditions leading to synthesize the controller $K_{p}$ under the $L_{2}$ norm bound are given in the following theorem 2 . Theorem 2: The tracking error $e_{p}(t)$ asymptotically converges to zero if there exists some matrices $P_{1}=P_{1}^{T} \geq 0$, $P_{3}$ and $K_{p}$ and a positive scalar $\bar{\gamma}$ such that the following LMI are satisfied for all $i=1,2, . ., r$

$$
\left(\begin{array}{ccccccc}
\Theta_{i} & * & * & * & 0 & 0 & 0 \\
P_{2}^{T} C_{i} & P_{2}+P_{2}^{T} & * & 0 & * & * & * \\
-B_{i}^{T} P_{1} & -D_{i}^{T} P_{2} & -\bar{\gamma} & 0 & 0 & 0 & 0 \\
P_{1} & 0 & 0 & -I & 0 & 0 & 0 \\
0 & B_{i} K_{p} & 0 & 0 & -I & 0 & 0 \\
0 & P_{2} & 0 & 0 & 0 & -I & 0 \\
0 & D_{i} K_{p} & 0 & 0 & 0 & 0 & -I
\end{array}\right)<0
$$

where $\Theta_{i}=P_{1} A_{i}+A_{i}^{T} P_{1}+I$.

Proof: Let us consider the following candidate quadratic Lyapunov function:

$$
V(e(t))=e^{T}(t) \bar{E} \bar{P} e(t)
$$

with

$$
\bar{E} \bar{P}=\bar{P}^{T} \bar{E} \geq 0
$$

we consider $\bar{P}=\left(\begin{array}{cc}P_{1} & 0 \\ 0 & P_{2}\end{array}\right)$. According to (29), one can find that $P_{1}=P_{1}^{T} \geq 0$. It is well known that the $L_{2}$-gain from $f(t)$ to $e(t)$ is bounded by $\gamma$ if [13]:

$$
\dot{e}^{T}(t) \overline{E P} e(t)+e^{T}(t) \overline{E P} \dot{e}(t)+e^{T}(t) e(t)-\gamma^{2} f^{T}(t) f(t)<0
$$

Considering (29) and substituting (26) in (30), one can obtain:

$$
\left(\begin{array}{cc}
\bar{P}^{T} \Gamma_{\mu}+\Gamma_{\mu}^{T} \bar{P}+\bar{E} & * \\
-\Lambda_{\mu}^{T} \bar{P} & -\gamma^{2}
\end{array}\right)<0
$$

The mathematical development of (31) with (26) and (29) leads to:

$$
\left(\begin{array}{ccc}
P_{1} A_{\mu}+A_{\mu}^{T} P_{1} & * & * \\
P_{2}^{T} C_{\mu}-K_{p}^{T} B_{\mu}^{T} P_{1} & -\left(K_{p}^{T} D_{\mu}^{T}-I\right) P_{2}-P_{2}^{T}\left(D_{\mu} K_{p}-I\right) & * \\
-B_{\mu}^{T} P_{1} & -D_{\mu}^{T} P_{2} & -\gamma^{2}
\end{array}\right)<0
$$

Applying Lemma 1 then Schur complement on (32), the sufficient LMI conditions proposed in theorem 2 holds. 